\title{
Effect of phonon-phonon interactions on localization
}

\author{
Abhishek Dhar \\ Raman Research Institute, Bangalore 560080, India \\ J.L. Lebowitz \\ Department of Mathematics and Physics, Rutgers University, Piscataway, NJ 08854
}

(Dated: October 26, 2018)

\begin{abstract}
We study the heat current $J$ in a classical one-dimensional disordered chain with onsite pinning and with ends connected to stochastic thermal reservoirs at different temperatures. In the absence of anharmonicity all modes are localized and there is a gap in the spectrum. Consequently $J$ decays exponentially with system size $N$. Using simulations we find that even a small amount of anharmonicity leads to a $J \sim 1 / N$ dependence, implying diffusive transport of energy.
\end{abstract}

PACS numbers: 44.10.+i, 05.60.Cd, 05.70.Ln

The effect of interactions between electrons or phonons on localization caused by disorder is a subject of both theoretical $1,1,2,3,4,5,6,6,8,8,9,10,11,12$ and experimental interest [13]. As is well known localization of eigenfunctions or of normal modes strongly affects transport in materials containing random impurities. Localization, first discovered in electronic systems by Anderson [14], has its strongest effect in one dimensions where any finite disorder makes all eigenstates localized [15] and one has an insulator. The presence of inelastic scattering, such as is caused by electron-phonon interactions, leads to hopping of electrons between localized states and gives rise to a finite conductivity. The question as to whether electron-electron interactions lead to a similar effect [2, 4] has attracted much attention recently but is still not fully understood [5, 6, 7, 8, 9].

In this paper we address the same question in the context of heat conduction by phonons and consider the effect that phonon-phonon interactions have on localization. In particular we investigate the effect of anharmonicities on the steady state transport of heat through a chain of oscillators with random masses. We focus on the case where the masses are subjected to an external pinning potential, in addition to nearest neighbor interactions. Pinning greatly enhances the difference between heat transport in a random chain with and without anharmonicity and thus is a good testing ground for the effect of anharmonicity on localization. We also discuss the unpinned case and comment on results from some earlier studies.

The Hamiltonian of our system has the form

$$
\begin{aligned}
H & =\sum_{l=1, N}\left[\frac{p_{l}^{2}}{2 m_{l}}+k_{o} \frac{x_{l}^{2}}{2}+\lambda \frac{x_{l}^{4}}{4}\right] \\
& +\sum_{l=1, N+1}\left[k \frac{\left(x_{l}-x_{l-1}\right)^{2}}{2}+\nu \frac{\left(x_{l}-x_{l-1}\right)^{4}}{4}\right]
\end{aligned}
$$

where $\left\{x_{l}, p_{l}\right\}$ denote the position and momenta of the particles and we set $x_{0}=x_{N+1}=0$. The masses $\left\{m_{l}\right\}$ are chosen independently from some distribution $p(m)$, e.g. one uniform in the interval $(m-\Delta, m+\Delta)$. The chain is connected at its ends to two heat baths at temperatures $T_{L}$ and $T_{R}$ respectively. The baths will be modeled by Ornstein-Uhlenbeck (Langevin white noise) reservoirs. The equations of motion of the chain are then given by:

$$
\begin{aligned}
m_{l} \ddot{x}_{l} & =-k_{o} x_{l}-l x_{l}^{3}-k\left(2 x_{l}-x_{l-1}-x_{l+1}\right) \\
& -\nu\left[\left(x_{l}-x_{l-1}\right)^{3}+\left(x_{l}-x_{l+1}\right)^{3}\right]-\gamma_{l} \dot{x}_{l}+\eta_{l}
\end{aligned}
$$

with $\eta_{l}=\eta_{L} \delta_{l, 1}+\eta_{R} \delta_{l, N}, \quad \gamma_{l}=\gamma\left(\delta_{l, 1}+\delta_{l, N}\right)$, and where the Gaussian noise terms satisfy the fluctuation dissipation relations $\left\langle\eta_{L}(t) \eta_{L}\left(t^{\prime}\right)\right\rangle=2 \gamma k_{B} T_{L} \delta\left(t-t^{\prime}\right)$, $\left\langle\eta_{R}(t) \eta_{R}\left(t^{\prime}\right)\right\rangle=2 \gamma k_{B} T_{R} \delta\left(t-t^{\prime}\right), k_{B}$ being Boltzmann's constant. It is known that this system has a unique stationary state [16].

Here we investigate the $N$ dependence of the heat current and the temperature profile, in the nonequilibrium stationary state (NESS) of this system, when $T_{L}>T_{R}$. The heat current from left to right is given by $\left\langle J_{N}\right\rangle=\sum_{l}\left\langle f_{l, l-1} \dot{x}_{l}\right\rangle /(N-1)$ where $f_{l, l-1}$ is the force exerted by the $(l-1)$ th particle on the $l$ th particle and $\langle\ldots\rangle$ denotes an average over the NESS. It follows from stationarity that each term in the sum, $l=2, \ldots, N$ is equal to $\left\langle J_{N}\right\rangle$.

Note that Eq.(2) is invariant under the transformation $T_{L, R} \rightarrow s T_{L, R},\left\{x_{l}\right\} \rightarrow\left\{s^{1 / 2} x_{l}\right\}$ and $(l, \nu) \rightarrow(l, \nu) / s$. This implies the scaling relation $\left\langle J_{N}\left(s T_{L}, s T_{R}, l, \nu\right)\right\rangle=$ $s\left\langle J_{N}\left(T_{L}, T_{R}, s l, s \nu\right)\right\rangle$. Thus the effect of changing nonlinearity could be equivalently studied by changing temperatures.

In the harmonic case, $\lambda=\nu=0$, the scaling gives $\left\langle J_{N}\right\rangle$ proportional to $\left(T_{L}-T_{R}\right)$. In this case the quadratic Hamiltonian can be written in the form $H_{0}=(1 / 2)\left[P^{T} M^{-1} P+X^{T} \Phi X\right]$ using matrix notation for the mass and force matrices. The stationary heat current, for any given realization of disorder is then given 
by the following expression [17, 18]:

$$
\begin{aligned}
& \left\langle J_{N}\right\rangle=\frac{k_{B}\left(T_{L}-T_{R}\right)}{\pi} \int_{-\infty}^{\infty} d \omega \mathcal{T}_{N}(\omega), \\
& \text { where } \quad \mathcal{T}_{N}(\omega)=\gamma^{2} \omega^{2}\left|G_{1 N}\right|^{2},
\end{aligned}
$$

with the matrix $G=\left[-\omega^{2} M+\Phi-\Sigma\right]^{-1}$ and $\Sigma_{l m}=$ $i \gamma \omega \delta_{l m}\left[\delta_{l 1}+\delta_{l N}\right]$.

The NESS in the harmonic chain without randomness, i.e $m_{l}=m$ can be solved exactly. It gives $\left\langle J_{N}\right\rangle \rightarrow c k_{B}\left(T_{L}-T_{R}\right)$, with $c>0$, for $N \rightarrow \infty$ [19, 20], i.e. the conductivity grows linearly with $N$.

The nature of $\left\langle J_{N}\right\rangle$ for the random harmonic chain without pinning was analyzed in much detail in 17,18 , 21, 22]. In [17] it was proved that, for an arbitrary nontrivial random mass distribution, $\left\langle J_{N}\right\rangle \rightarrow 0$, with $\left\langle J_{N}\right\rangle \geq N^{-3 / 2}$. The results in [17, 18, 21, 22] are based on the use of the Furstenberg theorem [23] first introduced into this problem by Matsuda and Ishii [24]. (In fact, Casher and Lebowitz use the $\left\langle J_{N}\right\rangle \rightarrow 0$ as $N \rightarrow \infty$ result to prove the absence of an absolutely continuous part of the spectrum for the random semi-infinite chain.) The fact that the random 1D system has no extended states is of course well-known. The mathematical proof given in 25] shows that such a chain has pure point spectrum, i.e. all the eigenfunctions are square integrable. The flux $\left\langle J_{N}\right\rangle$ in this chain is carried entirely by the long wave-length modes with frequencies $\omega \lesssim N^{-1 / 2}$ which "do not see" the randomness. More detailed results about this case can be found in $17,18,21,22]$. In particular, based on numerical evidence [18, 26] we believe that indeed $\left\langle J_{N}\right\rangle \sim N^{-3 / 2}$. We note however that $\left\langle J_{N}\right\rangle$ in the unpinned harmonic case with disorder depends on the particular type of heat bath [18]. For the Rubin model [22, 27], where the baths are semi-infinite ordered harmonic chains in equilibrium at temperatures $T_{L}\left(T_{R}\right)$ at the left (right) ends of the system and with no pinning anywhere, one gets $\left\langle J_{N}\right\rangle \sim N^{-1 / 2}$.

Considering now the random mass harmonic pinned case, $k_{o}>0$, one can show that $\left\langle J_{N}\right\rangle \sim e^{-c N}, c>0$. This follows from the fact that the spectrum of this chain now lies entirely in the interval $\left[\left(\frac{k_{o}}{m}\right)^{1 / 2},\left(\frac{k_{o}+2 k}{\underline{m}}\right)^{1 / 2}\right]$ where $\bar{m}(\underline{m})$ are the maximum (minimum) of the masses $\left\{m_{l}\right\}$. This means that there is a gap in the spectrum and Furstenberg's theorem implies that $\mathcal{T}_{N}(\omega) \lesssim$ $e^{-N \delta(\omega)}, \delta(\omega)>0$ for all $\omega$ in the allowed frequency range. An elementary calculation shows that $\mathcal{T}_{N}(\omega) \sim$ $e^{-N a}, a>0$ for $\omega$ outside the spectrum (true for both the ordered and disordered case). Hence, in the disordered harmonic pinned case, we will have asymptotically for almost all realizations of disorder

$$
-\frac{1}{N} \ln \left\langle J_{N}\right\rangle \sim c=\ell^{-1},
$$

where we can interpret $\ell$ as the largest localization length. A rough estimate of the value of $\ell$ can be obtained using known results for the unpinned case at small $\omega$. Substituting in these results the smallest allowed frequency $\omega_{m}^{2}=k_{o} / \bar{m}$ we get $\ell=12 \mathrm{~km} /\left(\omega_{m}^{2} \Delta^{2}\right)$ for masses chosen from a uniform distribution between $[m-\Delta, m+\Delta]$. This can be compared with the results from a numerical evaluation of the integral in Eq. (3) for the case with $k_{o}=k=1, m=1$ and $\Delta=0.2$. In Fig. (1) $(\lambda=0$ data $)$ we have plotted $N\left[\left\langle J_{N}\right\rangle\right]$, averaged over 100 disorder realizations, as a function of system size. From the data we find an exponential decay with $\ell \approx 200$ while our very rough estimate gives $\ell \approx 360$.

For the anharmonic chain there are no rigorous result about $\left\langle J_{N}\right\rangle$, even for the ordered case but the general expectation is that $\left\langle J_{N}\right\rangle \sim N^{\alpha-1}$ for the unpinned case. The actual value of $\alpha$ is a matter of some dispute with values ranging in the interval $\alpha=1 / 3-1 / 2[28,29,30]$. The most recent simulations found $\alpha=1 / 3$ [31].

A number of simulations for the disordered unpinned case with $\nu>0, k_{o}=\lambda=0$ show that anharmonicity in general tends to destroy localization [12]. In a recent work, Li et al 32 found a transition from $\alpha=0$ at small anharmonicity to $\alpha \sim 0.43$ at large anharmonicity. They did the simulations using a deterministic model for the reservoirs, namely Nose-Hoover thermostats. However this model of thermostats has been shown in [33] to be problematic for the harmonic case and these problems persist for small anharmonicity as well [34]. Preliminary simulations carried out for the unpinned disordered anharmonic case, with white noise Langevin heat baths, do not find evidence of a finite $\nu$ transition 35.

We now consider the pinned case $k=k_{o}=1, \lambda>$ $0, \nu=0$ when the system without randomness is much better understood. It is generally agreed that, in the absence of disorder, models with onsite pinning and anharmonicity show regular heat transport with $\left\langle J_{N}\right\rangle \sim 1 / N$ [36, 37]. This is proven rigorously for the case when a certain amount of stochasticity is added to the dynamics [38]. This model is also closer in spirit to charge transport by hopping in random media [14, 15] .

In our simulations with both disorder and anharmonicity we fixed the values of $k, T_{R},\langle m\rangle, \gamma$. We then measure time and distance in units of $(\langle m\rangle / k)^{1 / 2}$ and $\left(k_{B} T_{R} \gamma\right)^{1 / 2} / k$ respectively and set $k_{o} / k=1, T_{L} / T_{R}=2$. The only free parameters $\Delta$ and $\lambda$ are measured in units of $\langle m\rangle$ and $k^{3} /\left(k_{B} T_{R} \gamma\right)$ respectively (in the simulations we set $k=k_{o}=\langle m\rangle=\gamma=T_{R}=1$ ). In Fig. (11) we plot $N\left[\left\langle J_{N}\right\rangle\right]$ as a function of $N$ for a fixed disorder strength $\Delta=0.2$ and different values of anharmonicity $\lambda=0.004-1.0$. As can be seen from our data, there is a dramatic increase in the heat current on introduction of a small amount of anharmonicity and the system-size dependence goes from exponential decay to a $1 / N$ dependence implying diffusive transport. For smaller $\lambda$ the diffusive regime sets in at larger length scales. Similar results are obtained for the case with $\lambda=0, \nu>0$ and are shown in the inset of Fig. (1). 
The simulations were done by the velocity Verlet algorithm adapted for Langevin dynamics 39]. Equilibration times ranged from $10^{8}-2 \times 10^{8}$ time steps of step-size $0.005-0.01$ and steady state averages were taken over another $10^{8}-8 \times 10^{8}$ time steps. Equilibration times increase rapidly with decreasing $\lambda$ and with increasing $N$ and these cases required the longest runs. The error bars correspond to sample to sample fluctuations (other errors are smaller) and the number of samples varied from 10 for the small sizes to 3 for the largest $(N=4096)$. We find that the sample-sample fluctuations become smaller with increasing $N$. A measure of the relative strengths of anharmonicity and disorder is obtained by looking at the ratio of the energy scales $E_{a}=l\left\langle x^{4}\right\rangle / 4$ and $E_{d}=T \Delta / m$. For our parameters we estimate $\epsilon=E_{a} / E_{d} \approx 0.3 \lambda T / \Delta$ and for $T=\left(T_{L}+T_{R}\right) / 2=1.5$ this gives $\epsilon \approx 0.008$ for $\lambda=0.004$.

It follows from the scaling relation described earlier that $\left\langle J_{N}\right\rangle=\left(T_{L}-T_{R}\right) f\left(\lambda T_{L}, l T_{R}\right)$. Hence the conductivity, for $N \rightarrow \infty$ and $T_{L} \rightarrow T_{R}=T$, should depend only on $\lambda T, \kappa=\kappa(l T)$. For $T_{L}>T_{R}$ the temperature and hence the conductivity varies across the chain, we therefore measure the effective thermal conductivity $\kappa_{e f f}=N\left[<J_{N}>\right]$, evaluated at large $N$ (boundary temperature jumps are negligible). For small $\lambda$ we find $\kappa_{\text {eff }} \sim(l T)^{a}$ with $a<1$. Assuming that this is also the behaviour of $\kappa$ this implies, using the fact that $\kappa d T / d y=$ constant, that the temperature profile is given by $T(y)=\left[T_{L}^{a+1}(1-y)+T_{R}^{a+1} y\right]^{1 /(a+1)}$ where $y$ is the distance, scaled by $N$, from the left end of the chain. We now look at the local temperature profiles in the NESS obtained in the simulations. The local temperature at the $i$ th site is defined by $T_{i}=m_{i}\left\langle\dot{x}_{i}^{2}\right\rangle$. The temperature profiles for $l=0.1$ for a single sample, with and without disorder, are plotted in Fig. (2). The plots (a),(b) correspond respectively to averaging over $8 \times 10^{8}$ and $32 \times 10^{8}$ time steps with $d t=0.005$ and show that the profiles are reasonably converged.The noise amplitude does not decrease much on increased averaging. We find that the temperature profile, for the disordered case, is consistent with the predicted form with $a \sim 1 / 2$ (see however below).

For the case without disorder we find a temperature profile consistent with a $1 / T^{2}$ (thus $a=-2$ ) dependence for the conductivity. The $\kappa(T) \sim 1 / T^{2}$ dependence has been predicted from recent kinetic theory calculations and should apply for small nonlinearity [37]. The inset in Fig. (2) shows temperature profiles for the disordered chain with a smaller and a larger value of $\lambda$. We find that these profiles have some amount of structure, possibly reflecting the local mass distribution, and vary appreciably from sample to sample.

Discussion: We have studied heat conduction in a disordered pinned anharmonic chain. We find that introduction of a small amount of phonon-phonon interactions in the disordered harmonic chain leads to diffusive energy

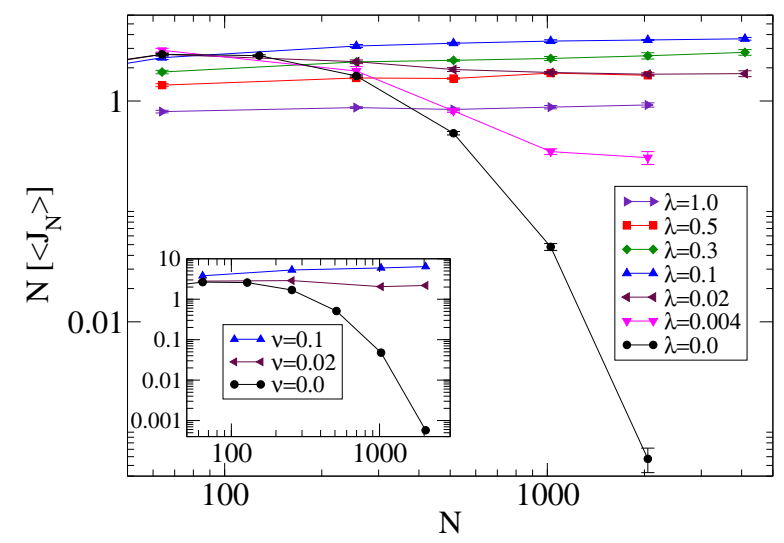

FIG. 1: Plot of the disordered averaged heat current $\left[\left\langle J_{N}\right\rangle\right]$ multiplied by $N$ as a function of $N$ for different values of $\lambda$. The inset shows results obtained for the case with interparticle anharmonicity.

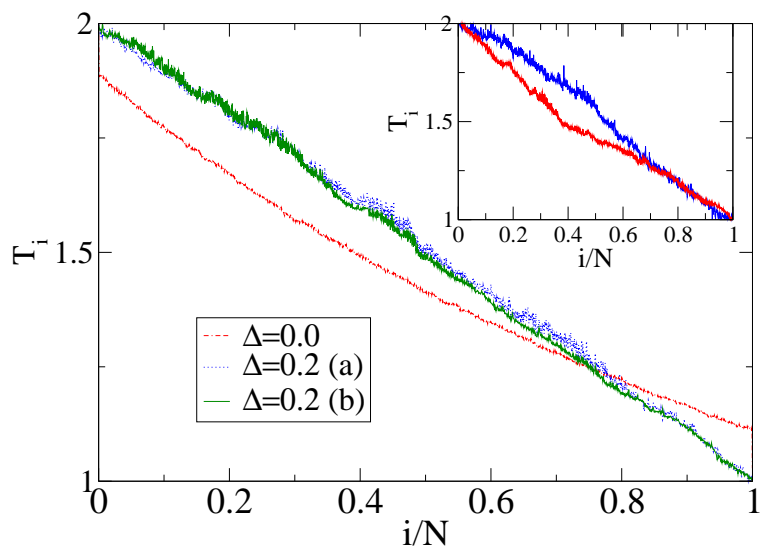

FIG. 2: The temperature profile for an anharmonic chain with $\lambda=0.1$ and $N=2048$ is shown for both the ordered and disordered cases. The inset shows the temperature profiles for a smaller value of anharmoncity $\lambda=0.02$ (upper curve) and a larger value $\lambda=0.5$. In all disordered cases $\Delta=0.2$.

transfer, i.e. the insulating chain becomes a normal heat conductor. We do not find evidence of the existence of a finite critical value of anharmonicity required for this transition. For small values of anharmonicity it is necessary to go to larger system sizes to see the transition from insulating to diffusive. Hence a transition to a localized phase at a very small value of anharmonicity is possible and would be difficult to observe in simulations. Even assuming that no such transition occurs, the limiting behavior of $\kappa(\lambda T)$ for $(\lambda T) \rightarrow 0$ cannot be obtained from our data. A. Pal and D. Huse [40] have investigated this type of question for a chain of classical spins. Their system corresponds very roughly to the case where $k=\lambda=0$ and so the interaction between particles on different sites only comes via $\nu$ : they find a diffusivity which behaves like $e^{-c / \nu}$. We remark that for the or- 
dered harmonic chain letting $k \rightarrow 0$ gives $\left\langle J_{N}\right\rangle \sim k$ for the unpinned case and $\left\langle J_{N}\right\rangle \sim k^{2}$ for the pinned case.

We note that in the ordered chain nonlinearity leads to scattering of otherwise freely moving phonons thereby changing the system from a super-heat conductor to a normal one. By contrast, in the disordered chain the nonlinearity appears to produce effective extended states which lead to diffusive transfer of energy between local regions. How exactly this occurs is not clear.

The diffusive transport observed in our disordered open system seems to differ from recent numerical studies of spreading of localized energy pulses in disordered nonlinear lattices which suggest sub-diffusion [8] or even absence of diffusion [9]. To understand this we have also performed some studies on our system without heat baths. From these we find that while a heat pulse introduced at one end of a long chain does indeed not propagate, a periodic driving at one end leads to significant energy transmission. This suggests that an isolated pulse behaves differently from a continuously driven or open system [41].

Finally we note that experimental measurements of heat conduction in one-dimensional systems are now becoming possible [42, 43, 44]. Interesting measurable effects which our study suggests are the effect of temperature and system-size on heat conduction in disordered wires. Both of these enhance the effect of nonlinearity and lead to diffusive transport.

We thank M. Aizenmann, E. Lieb, J. Lukkarinen, V. Oganesyan, T. Spencer, H. Spohn and especially D. Huse for useful discussions. AD thanks K. Saito and B. S. Shastry for discussions. Research supported in part by NSF grant 0442066 and AFOSR grant AF-FA9550-04.

[1] L. Fleishman and P. W. Anderson, Phys. Rev. B 21, 2366 (1980).

[2] L. Fleishman, D. C. Licciardello and P. W. Anderson, Phys. Rev. Lett. 40, 1340(1978).

[3] T. Giamarchi and H.J. Schultz, Phys. Rev. B37, 325 (1988).

[4] T. Nattermann, T. Giamarchi, and P. Le Doussal, Phys. Rev. Lett. 91, 056603 (2003).

[5] I. V. Gornyi, A. D. Mirlin, and D. G. Polyakov, Phys. Rev. Lett. 95, 206603 (2005).

[6] D. M. Basko, I. L. Aleiner and B. L. Altshuler, Ann. Phys. (N.Y.) 321, 1126 (2006).

[7] V. Oganesyan, D. A. Huse, Phys. Rev. B 75, 155111 (2007).

[8] A. S. Pikovsky and D.L.Shepelyansky, Phys. Rev. Lett. 100, 094101 (2008).

[9] G. Kopidakis et al, Phys. Rev. Lett. 100, 084103 (2008).

[10] P. H. Song and D. L. Shepelyansky, Phys. Rev. B 61, 15546 (2000)

[11] R. T. Scalettar, G. G. Batrouni, G. T. Zimanyi, Phys. Rev. Lett. 66, 3144 (1991).

[12] D. N. Payton, M. Rich and W. M. Visscher, Phys. Rev. 160, 706 (1967); R. Bourbonnais and R. Maynard, Phys.
Rev. Lett. 64, 1397 (1990); K. A. Snyder and T. R. Kirkpatrick, Phys. Rev. B 73, 134204 (2006).

[13] Yu. B. Khavin et al., Phys. Rev. B 58, 8009 (1998); M. E. Gershenson et al., Phys. Rev. Lett. 79, 725 (1997); S. Y. Hsu and J. M. Valles, Phys. Rev. Lett. 74, 2331 (1995); F. W. van Keuls et al., Phys. Rev. B 56, 13263 (1997); G. M. Minkov et al., ibid. 65, 235322 (2002).

[14] P. W. Anderson, Phys. Rev. 109, 1492 (1958).

[15] N. F. Mott and W. D. Twose, Adv. Phys. 10, 107 (1961); R. E. Borland, Proc. R. Soc. London, Ser. A 274, 529 (1963).

[16] J.-P. Eckmann, C.-A. Pillet, L. Rey-Bellet, Comm. Math. Phys. 201, 657 (1999).

[17] A. Casher and J. L. Lebowitz, J. Math. Phys. 12, 1701 (1971).

[18] A. Dhar, Phys. Rev. Lett. 86, 5882 (2001).

[19] Z. Rieder, J. L. Lebowitz and E. Lieb, J. Math. Phys. 8, 1073 (1967).

[20] H. Nakazawa, Prog. Theor. Phys. Suppl. 45, 231 (1970).

[21] A. J. O'Connor and J. L. Lebowitz, J. Math. Phys. 15, 692 (1974).

[22] R. Rubin and W. Greer, J. Math. Phys. 12, 1686 (1971).

[23] H. Furstenberg, Trans. Am. Math. Soc. 108, 377 (1963).

[24] H. Matsuda and K. Ishii, Suppl. Prog. Theor. Phys. 45, $56(1970)$

[25] I. Ya. Gol'dshtein, S. A. Molchanov and L. A. Pastur, Funct. Anal. Appl. 11, 1 (1977).

[26] V. N. Likhachev et al, Phys. Rev. E 73, 016701 (2006).

[27] T. Verheggen, Comm. Math. Phys. 68, 69 (1979).

[28] O. Narayan and S. Ramaswamy, Phys. Rev. Lett. 89, 200601 (2002).

[29] S. Lepri, R.Livi and A. Politi, Phys. Rev. E 68, 067102 (2003); J.S. Wang and B.Li, Phys. Rev. Lett. 92, 074302 (2004).

[30] G. Basile, C. Bernardin and S. Olla, Phys. Rev. Lett. 96, 204303 (2006).

[31] T. Mai, A. Dhar and O. Narayan, Phys. Rev. Lett. 98, 184301 (2007).

[32] B. Li, H. Zhao and Bambi Hu, Phys. Rev. Lett. 86, 63 (2001).

[33] A. Dhar, Phys. Rev. Lett. 87, 069401 (2001).

[34] H. A. Posch, W. G. Hoover, and F. J. Vesely, Phys. Rev. A 33, 4253 (1986); A. Dhar, unpublished notes.

[35] A.Dhar, K.Saito and J.L.Lebowitz, work in progress.

[36] G. Casati et al., Phys. Rev. Lett. 52, 1861 (1984); T. Prosen and M. Robnik, J. Phys. A 25, 3449 (1992); B. Hu, B. Li and H. Zhao, Phys. Rev. E 57, 2992 (1998); K. Aoki and D. Kusnezov, Phys. Lett. A 265, 250 (2000); B. Hu.,B. Li and H. Zhao, Phys. Rev. E 61, 3828 (2000);

[37] K. Aoki, J. Lukkarinen and H. Spohn, J. Stat. Phys. 124, 1105 (2006).

[38] F. Bonetto, J.L. Lebowitz, J. Lukkarinen and S. Olla, in preparation.

[39] M. P. Allen and D. L. Tildesley, Computer Simulations of Liquids (Clarendon, Oxford, 1987).

[40] A. Pal and D. Huse, private communication.

[41] H. Zhao, Phys. Rev. Lett. 96, 140602 (2006).

[42] D. E. Angelescu, M. C. Cross and M. L. Roukes, Superlatt. Microstruct. 23, 673 (1998).

[43] Schwab et al., Nature 404, 974 (2000); H. -Y. Chiu et al., Phys. Rev. Lett. 95, 226101 (2005).

[44] Z. Wang et. al., Science 317, 787 (2007); A. Nitzan, Science 317, 759 (2007). 